\title{
Loxoscelismo cutáneo predominantemente edematoso, a propósito de un caso
}

\author{
Paula de la Barra, Verónica Vial, Yenis Labraña, Ana María Álvarez y Helena Seguel
}

\section{Cutaneous loxoscelism mainly edematous, a case report}

In Chile, loxoscelism is caused by the bite of the Loxosceles laeta spider. The clinical presentation has two different forms: cutaneous loxoscelism (CL) and vicero-cutaneous loxoscelism, which is less frequent. Cutaneous loxoscelism includes an uncommon clinical variation (4\%), called CL with edematous predominance (CLEP). We present a 5-year-old patient with sudden pain and edema on his right eyelid associated with fever, which progressed rapidly involving the right hemifacial area, frontal region, and left eyelid. Angioedema and pre-orbital cellulitis were discarded and CLEP was suspect. Cutaneous loxoscelism with an edematous predominance is self-limited, benign and with little or no necrotic injury due to the edema, which dilutes the toxin-induced enzymatic process causing necrosis. As in the reported cases it usually responds well to medical treatment and does not cause visceral involvement.

Key words: Cutaneous loxoscelism, edema, Loxosceles laeta.

Palabras clave: Loxoscelismo cutáneo, edema, Loxosceles laeta.

\section{Introducción}

$\mathrm{E}$ 1 loxoscelismo es un cuadro tóxico producido por la mordedura de la araña del género Loxosceles, que ha sido registrado en la mayoría de los países americanos. En Chile es producido por la especie Loxosceles laeta, conocida como "araña de rincón". Ésta se adapta fácilmente a las condiciones domiciliarias, escondiéndose principalmente detrás de cuadros, muebles, ropa y zapatos; siempre protegida de la luz directa. El cuadro clínico puede presentarse bajo dos formas: loxoscelismo cutáneo (LC), en 81 a 96\% de los casos, y loxoscelismo cutáneo-visceral (LCV) o sistémico, en 7,3 a $18 \%$ de los casos ${ }^{1,2}$. Dentro del loxoscelismo cutáneo se encuentra una variante poco común ( $4 \%$ de los casos) conocida como loxoscelismo cutáneo predominantemente edematoso (LCPE) $)^{3}$.

\section{Caso clínico}

Preescolar de 5 años sin antecedentes mórbidos. Consultó en el Servicio de Urgencia por un súbito inicio de aumento de volumen palpebral derecho, doloroso, con imposibilidad de apertura ocular, asociado a fiebre hasta $39^{\circ} \mathrm{C}$. Evolucionó con un aumento de volumen rápidamente progresivo, extendiéndose hacia la hemicara derecha, región frontal y párpado izquierdo, impidiendo la apertura de ambos ojos (Figura 1). No presentó cambios de voz ni dificultad respiratoria. No se evidenció puerta de entrada ni cambios de coloración en la piel, sólo destacaba la presencia de tres pequeñas vesículas en el párpado superior derecho. Dentro de los exámenes generales tenía un hemograma con leucocitosis leve, sin anemia y plaquetas normales, sin aumento de PCR. Tomografía axial computarizada (TAC) de órbita normal. Durante su hospitalización fue evaluado por un equipo multidisciplinario (infectología, otorrinolaringología y oftalmología). Se descartó un angioedema y una celulitis periorbitaria. Finalmente se realizó el diagnóstico de un LCPE. Se inició tratamiento con penicilina, hidrocortisona y clorfenamina i.v. Evolucionó con persistencia del edema facial, con una lenta resolución a partir del octavo día (Figuras 2, 3, 4). Mantuvo una diuresis normal, con un examen de orina normal, sin hemoglobinuria, y con leve anemia en el hemograma.

\section{Discusión}

Las arañas del género Loxosceles son de distribución mundial y viven cercanas o dentro de las viviendas. En Sudamérica existen más de 30 especies de Loxosceles, siendo L. laeta la de mayor importancia. El cuadro clínico causado por la mordedura de las diferentes arañas de esta especie se conoce como loxoscelismo. En algunos países es un problema de salud pública como sucede en algunas regiones de Argentina, Brasil, Perú y Chile. En el caso de este último y de acuerdo a los datos publicados, en el año 2005 el Centro de Información Toxicológica de la
Universidad de Chile, Facultad de Medicina.

Programa de Especialidad en Pediatría (PD, HS).

Calera de Tango, Chile.

Médico general CES, Los Bajos San Agustín (VV).

Hospital San Juan de Dios, Santiago, Chile.

Servicio de Infectología (YL, AMA).

Recibido: 11 de noviembre de 2014 Aceptado: 17 de abril de 2015

Correspondencia a:

Paula de la Barra Cánepa pola.aidesu@gmail.com 


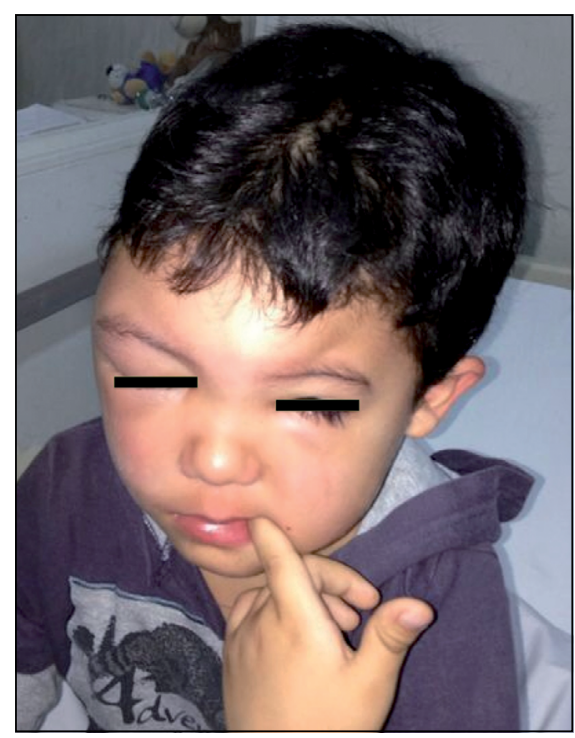

Figura 1. Evolución día 1: Edema palpebral bilateral, mayor a derecha.

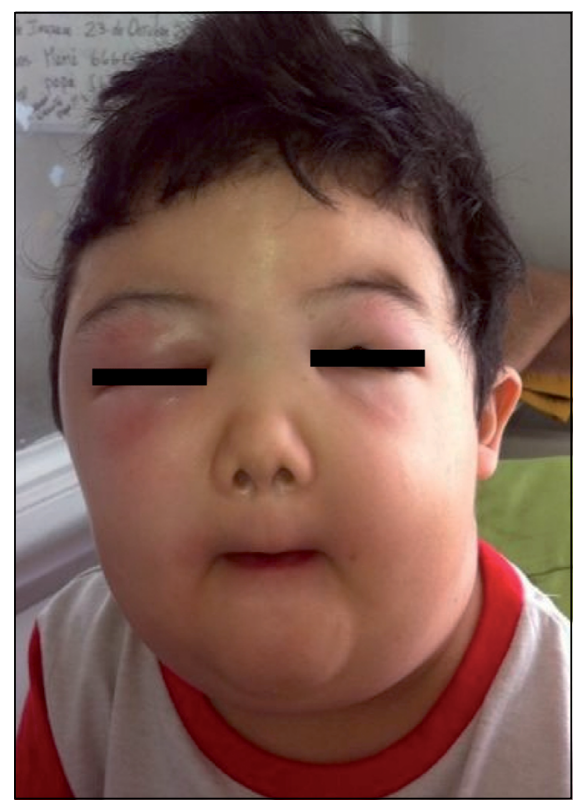

Figura 3. Evolución día 5 : Persistencia de edema facial, palpebral bilateral que impide apertura ocular.

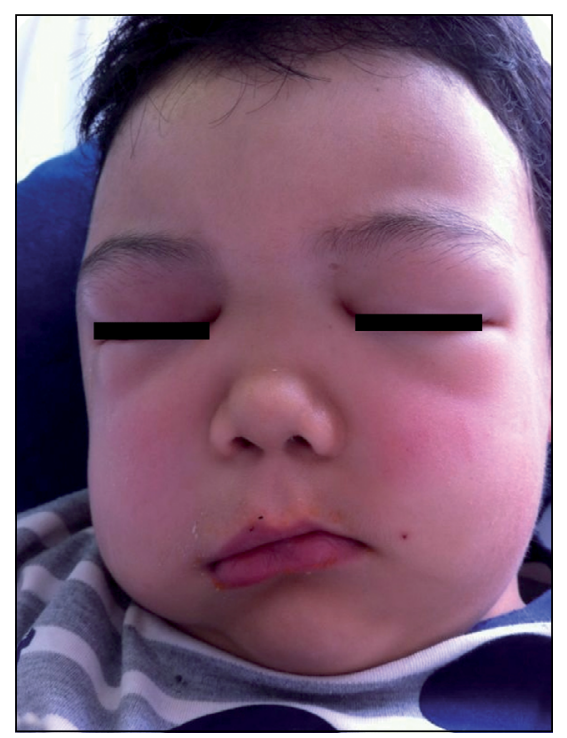

Figura 2. Evolución día 3: Gran edema facial, principalmente a nivel palpebral bilateral que impide apertura ocular.
Figura 4. Evolución día 8: Comienzo de regresión de edema facial, disminución de edema palpebral, logra apertura ocular sin problemas.
Universidad Católica de Chile atendió 2.831 llamados sobre pacientes con sospecha de loxoscelismo, de los que se confirmaron $287^{4,5}$.

Loxosceles laeta en Chile específicamente se ha descrito desde la Región de Iquique a la del Bío-Bío. Las arañas del género Loxosceles son tímidas, solitarias y sólo muerden si se les molesta.

Loxosceles laeta es una araña pequeña que mide aproximadamente $1 \mathrm{a} 4,5 \mathrm{~cm}$ de longitud (incluyendo las patas), es de color café y tiene en el dorso del cefalotórax una mancha en forma de violín invertido, que puede variar de tonalidad de café oscura a amarilla. Posee característicamente tres pares de ojos, lo que es muy importante para su identificación ${ }^{1}$.

La mayoría de los accidentes ocurre en la noche, mientras la persona duerme, o en el momento de vestirse en la mañana. Aunque el accidente puede ocurrir en cualquier estación del año, su mayor frecuencia es en verano y primavera ${ }^{6,7}$.

El veneno contiene hialuronidasa, ARNasa, ADNasa, fosfatasa alcalina, lipasas y esfingomielinasa $\mathrm{D}^{8}$. Tiene propiedad necrosante, hemolítica, vasculítica y coagulan- 
te. En la piel provoca áreas de vasoconstricción y otras de hemorragia, que llevan rápidamente a la isquemia local y a la formación de una placa gangrenosa. Si el veneno alcanza la circulación sistémica, ejerce un gran poder hemolítico, que es el aspecto central del LCV ${ }^{9}$.

El LC es un cuadro autolimitado, benigno, asociado a prurito, edema y eritema dentro de las primeras 6 a $8 \mathrm{~h}$ de la mordedura. Progresa a un halo de vasoconstricción e isquemia que se extiende alrededor de la lesión conocida como placa livedoide, la que en algunos casos evoluciona a una úlcera necrótica con un hundimiento central.

El LCV se caracteriza porque, además de las manifestaciones cutáneas, aparecen en las primeras $24 \mathrm{~h}^{10}$ intensos síntomas y signos sistémicos como fiebre alta y mantenida, calofríos, decaimiento, cefalea, náuseas, vómitos y mialgias. El evento más importante es la hemólisis intravascular masiva, que comienza a hacerse evidente a partir de 6 a 12 h de ocurrida la mordedura. Se produce una anemia aguda, ictericia, palidez, cianosis, hipotensión arterial, hemoglobinuria y hematuria, y compromiso de conciencia de grado variable. Los pacientes con hemólisis masiva pueden desarrollar una necrosis tubular aguda manifestada por insuficiencia renal, con oliguria o anuria o signos de insuficiencia cardíaca. Tiene una letalidad entre 5 y $20 \%{ }^{10}$.

En el LCPE no está presente la lesión necrótica o es muy insignificante, predomina el edema, especialmente cuando la mordedura es facial. El edema abortaría el proceso necrótico al diluir el proceso enzimático producido por el veneno. Es de buen pronóstico ya que tiene buena respuesta a tratamiento médico y en los pocos casos reportados, ninguno presentó compromiso visceral ${ }^{3}$.

El edema se caracteriza por ser rosado brillante, con aumento de la temperatura local, doloroso, indurado, sin dejar huella a la presión y no se acompaña de adenopatía regional $^{3}$. Según Schenone, pareciera haber una relación en el sitio comprometido y la forma edematosa ya que la mayoría de los casos de LCPE son mordeduras en cara, cuello y sectores proximales a las extremidades ${ }^{2}$.

El LCPE es de difícil diagnóstico, ya que es poco frecuente y no es el primer diagnóstico que pensamos. Dentro de los diagnósticos diferenciales se encuentran la picadura facial de insectos hematófagos, picadura de abeja, enfermedad de Chagas con puerta de entrada facial, angioedema y celulitis periorbitaria, entre otros.

Lo que determina el tipo de presentación clínica del cuadro es la susceptibilidad individual de cada paciente, independiente de la cantidad de veneno inoculado. Es importante destacar que en los niños el cuadro de LCV es siempre más grave, presentan mayor frecuencia de coagulación intravascular diseminada, insuficiencia renal aguda, falla multiorgánica y mayor letalidad, la cual va desde 17 a $30 \%$, por lo que se recomienda la hospitalización en todos los casos de loxoscelismo en pediatría ${ }^{5-8}$.
La producción del LCV y su eventual letalidad no están relacionadas con el sitio de mordedura, la extensión de la lesión local ni la época del año en que se produjo el accidente. La aparición del compromiso visceral es determinante en el pronóstico. $\mathrm{Si}$ dicho compromiso no se desarrolla en las primeras $24 \mathrm{~h}$, existen muchas probabilidades que se trate de un LC; pasadas las $48 \mathrm{~h}$ de evolución, el pronóstico es bueno ${ }^{7}$.

El diagnóstico del loxoscelismo es difícil debido al desconocimiento de la población general de los signos y síntomas iniciales, lo que lleva a una consulta tardía. El diagnóstico es habitualmente presuntivo, basado en la información epidemiológica y la evolución del cuadro clínico. La minoría de los pacientes $(\sim 10 \%)$ trae al agente biológico para su identificación directa 9 .

Es muy importante siempre indagar si el relato que da el paciente corresponde a las características típicas del accidente de esta araña. Las mordeduras ocurren generalmente dentro del domicilio, en zonas urbanas y por las mañanas, cuando las personas se están vistiendo o cuando están limpiando áreas dentro de la casa que no se remueven con frecuencia. También se reportan mordeduras por la noche mientras se duerme. Cuando la persona está consciente puede referir un dolor súbito lancinante una sola vez o bien la mordedura puede pasar desapercibida ${ }^{10}$.

Las zonas más afectadas son los miembros superiores e inferiores, el rostro, el cuello y el tronco ${ }^{10}$.

No existen exámenes de laboratorio que confirmen por sí solo el diagnóstico, pero en caso de sospecha es importante solicitar: hemograma en busca de hemólisis, orina completa en busca de hemoglobinuria y hematuria, creatininemia para descartar una insuficiencia renal y tiempo de protrombina y tromboplastina, para descartar una coagulopatía9.

La concurrencia precoz del paciente a centros asistenciales y el diagnóstico oportuno son cruciales. El tratamiento consiste en la administración parenteral de antihistamínicos y/o corticoesteroides por su acción antiinflamatoria; el veneno produce alteraciones de la mucosa gástrica e intestinal, por lo que su administración oral no está recomendada ${ }^{6}$.

Ante la sospecha de la mordedura de una araña de rincón siempre se debe consultar en el Servicio de Urgencia más cercano para la primera evaluación del paciente y posterior control, realizar una curación frecuente de la herida, mantener elevada e inmovilizada la extremidad afectada, profilaxis antitetánica, analgésicos y antihistamínicos. En caso de haber compromiso visceral es importante la hidratación, transfusión sanguínea, diuréticos e incluso diálisis si es necesario. Si se comprueba hemoglobinuria se debe alcalinizar la orina con bicarbonato.

En E. U.A. las lesiones necróticas se tratan con antimicrobianos para prevenir infecciones. En países en que las 
infecciones secundarias no son frecuentes, como en Chile, no se recomienda el uso de antibacterianos como profilaxis y sólo se indica ante la evidencia de sobreinfección.

Los corticoesteroides se han usado precozmente en niños para prevenir la hemólisis y el daño renal. Sin embargo, éstos no inactivan el veneno, no previenen el daño primario ni la necrosis cutánea ${ }^{4}$. Los corticoesteroides, actualmente en uso, disminuirían la respuesta inflamatoria local en los casos graves con compromiso sistémico. Se utiliza prednisona $0,5-1 \mathrm{mg} / \mathrm{kg}$ /día por $5-7$ días. Se ha utilizado dapsona, por la inhibición de la migración de polimorfonucleares en el sitio de la mordedura, lo que es esencial para prevenir las lesiones dermonecróticas. No está indicado en loxoscelismo visceral ${ }^{4,11}$. También se ha usado el oxígeno hiperbárico, que mejoraría la vascularización de la zona afectada. Sin embargo, ninguno de estos tratamientos posee eficacia clínica avalada por estudios controlados prospectivos en seres humanos ${ }^{11}$.

La medida específica es el uso del anti-veneno. Se trata de un antisuero de origen equino que se realiza a partir del veneno de arañas del género Loxosceles. Su mayor eficacia se ha observado dentro de las primeras $12 \mathrm{~h}$. El plazo máximo de utilización del suero es controvertido: en la fase aguda se puede indicar hasta las $36 \mathrm{~h}$ del accidente y en la forma cutáneo-visceral se indica siempre que se constate hemólisis ${ }^{11}$. Existen varios estudios que demuestran su beneficio en modelos animales cuando el veneno es administrado en las primeras horas, tanto en su actividad hemolítica como dermo-necrótica; sin embargo, no hay evidencia sólida y suficiente que recomiende el uso de suero anti-loxosceles en humanos ${ }^{10}$. Los preparados específicos de suero anti-loxosceles que existen en Chile son traídos de Perú. Se ha sostenido que éstos son más efectivos y se mencionan casos en que fueron utilizados exitosamente aun en forma tardía, a los tres días de ocurrida la mordedura ${ }^{12}$.

En los últimos estudios experimentales de Dias-Lopes y Felicori y cols., muestran la utilidad potencial de los anticuerpos monoclonales para los enfoques terapéuticos y de inmunodiagnóstico en $\mathrm{LC}^{13}$.

Para la prevención de la mordedura es importante el aseo, orden e higiene en todos los recintos, rincones, detrás de muebles, libros y cuadros, separar las camas de los muros, no colgar ropas ni toallas en los muros, revisar y sacudir la ropa antes de vestirse, evitar que los niños jueguen en el interior de closets y usar aracnicidas adecuados. Además es importante educar a la población en el reconocimiento del arácnido y en los síntomas que produce la mordedura. Si hay sospecha de haber sido mordido se debe tratar de capturar la araña para su identificación.

El diagnóstico, como se mencionó anteriormente, es difícil y se realiza según la epidemiología local y evolu- ción clínica. En este caso no se evidenció una puerta de entrada clara, placa necrótica ni compromiso visceral. Lo que llevó al diagnóstico fue el antecedente que el paciente reportó un inicio súbito de dolor facial nocturno y un gran edema facial rosado brillante, indurado y doloroso que fue en aumento progresivo y su buena respuesta al tratamiento médico. Se descartó una celulitis periorbitaria con una TAC de órbita normal y sin ascenso de parámetros inflamatorios. Por otro lado, un angioedema era poco probable por la lenta resolución del compromiso facial, ya que éste generalmente dura hasta 3 días pese al tratamiento con corticoesteroides y antihistamínicos sistémicos. En nuestro caso la resolución comenzó recién al octavo día, además no presentó compromiso de labios, lengua, ni dificultad respiratoria, ni signos de compromiso sistémico (anafilaxia).

Con respecto a las lesiones vesiculares sobre el párpado superior derecho, se descartó un herpes simplex por la ausencia de ardor y prurito previo a la aparición de las lesiones; además no tenía antecedentes de lesiones herpéticas. También se descartó herpes zoster ya que el paciente no tenía el antecedente de varicela, las lesiones no eran metaméricas y no hubo progresión de éstas, desapareciendo al segundo día.

En Chile, el loxoscelismo constituye un problema importante de salud pública, donde no existen intervenciones terapéuticas estándares. Comunicamos este caso por tratarse de una presentación poco común y mal diagnosticada de la mordedura de la araña de rincón, con escasos reportes en la literatura especializada.

\section{Resumen}

El loxoscelismo en Chile es un cuadro producido por la mordedura de la araña Loxosceles laeta. Las formas de presentación son: loxoscelismo cutáneo (LC) y loxoscelismo cutáneo-visceral (LCV), el último menos frecuente. Dentro del LC existe una variante poco común $(4 \%)$ conocida como loxoscelismo cutáneo predominantemente edematoso (LCPE). Nuestro caso es un paciente de 5 años que consultó por cuadro de inicio súbito de dolor y edema palpebral derecho, asociado a fiebre el cual evolucionó con rápida progresión extendiéndose en la hemicara derecha, región frontal y párpado izquierdo. Se descartó un angioedema y una celulitis periorbitaria, sospechándose un LCPE. Se manejó con hidrocortisona y clorfenamina. El LCPE es un cuadro benigno, autolimitado, en que no está presente la lesión necrótica o ésta es insignificante. Predomina el edema, el cual abortaría la necrosis al diluir el proceso enzimático producido por el veneno. Tiene buena respuesta al tratamiento médico, con ausencia de compromiso visceral. 


\section{Referencias bibliográficas}

1.- Ríos J C, Pérez M, Sánchez P, Bettini M, Mieres J J, Paris E. Caracterización clínicoepidemiológica telefónica de la mordedura por araña de rincón, en un centro de información toxicológica de Chile, durante el año 2005. Rev Med Chile 2007; 135: 1160-5.

2.- Schenone F H. Cuadros tóxicos producidos por mordeduras de araña en Chile: latrodectismo y loxoscelismo. Rev Med Chile 2003; 131: 437-44.

3.- Schenone H. Loxocelismo cutáneo de predominio edematoso. Bol Chil Parasitol 1998; 53: 78-83.

4.- Manríquez M, Silva S. Loxoscelismo cutáneo y cutáneo-visceral: revisión sistemática. Rev Chilena Infectol 2009; 26: 420-32.

5.- Baldovino R, Moreira N, Fernández A, Ferré A, Guerra M, Jaureguiberry J, et al. Loxoscelismo cutáneo: A propósito de un caso clínico. Arch Pediatría Urug 2012; 83: 273-7.

6.- Schenone H. A propósito del loxoscelismo en Chile. Rev Med Chile 2004; 132: 121-2.

7.- Schenone F H, Rubio A S, Saavedra U T, Rojas A. Loxoscelismo en pediatría: Región Metropolitana, Chile. Rev Chil Pediatr 2001; 72: 100-9.

8.- Levin C, Rozemman D, Sakran W, Halevy R, Peleg S, Koren A. Severe thrombocytopenia and dermonecrosis after Loxosceles spider bite in a 3-year-old child. J Pediatr 2013; 163 : 1228.e1.

9.- Ministerio de Salud, Chile. Loxoscelismo. Disponible: http://epi.minsal.cl/epi/html/ Actualidad/Nacional/loxocelismo1.pdf (accedido: 12 de septiembre de 2014).

10.- Ticona C A, Sánchez C C. Loxoscelismo: Evaluación Clínica, Tratamiento y Prevención 2006; 5: 1-5.

11.- Cabrerizo S, Docampo P C, Cari C, Ortiz de Rozas M, Díaz M, de Roodt A, et al. Loxoscelismo: epidemiología y clínica de una patología endémica en el país. Arch Argent Pediat 2009; 107: 152-9.

12.- Ministerio de Salud, Chile. División de Planificación y Presupuesto Departamento de Calidad de Prestadores Unidad de Evaluación de Tecnologías de Salud. Efectividad del suero anti-loxosceles: síntesis de evidencia. Agosto de 2004. Disponible en: http:// bases.bireme.br/cgi-bin/wxislind.exe/iah/ online/?IsisScript $=$ iah/iah.xis\&src $=$ google $\&$ base $=$ LILACS\&lang $=$ \&\&nextAction $=1 n k \&$ expr Search=665370\&indexSearch=ID (accedido: 14 de abril de 2015).

13.- Dias-Lopes C, Felicori L, Rubrecht L, Cobo S, Molina L, Nguyen C, et al. Generation and molecular characterization of a monoclonal antibody reactive with conserved epitope in sphingomyelinases D from Loxosceles spider venoms. Vaccine 2014; 32: 2086-92. 